\title{
BMJ
}

\section{Cryotherapy versus salicylic acid for the treatment of plantar warts (verrucae): a randomised controlled trial}

\author{
Sarah Cockayne, research fellow, ${ }^{1}$ Catherine Hewitt, statistician, ${ }^{1}$ Kate Hicks, research fellow, ${ }^{1}$ Shalmini \\ Jayakody, trial statistician, ${ }^{1}$ Arthur Ricky Kang'ombe, trial statistician, ${ }^{1}$ Eugena Stamuli, trial health economist, ${ }^{1}$ \\ Gwen Turner, trials support officer, ${ }^{1}$ Kim Thomas, associate professor, ${ }^{2}$ Mike Curran, senior lecturer podiatry, ${ }^{3}$ \\ Gary Denby, research assistant, ${ }^{3}$ Farina Hashmi, senior lecturer, ${ }^{4}$ Caroline McIntosh, senior lecturer, ${ }^{5}$ Nichola \\ McLarnon, senior lecturer, ${ }^{6}$ David Torgerson, professor, director of York Trials Unit," lan Watt, professor, \\ primary care ${ }^{1,7}$ on behalf of the EVerT Team
}

${ }^{1}$ Department of Health Sciences, York Trials Unit, University of York, York Y010 5DD, UK

${ }^{2}$ Centre of Evidence Based Dermatology, University of Nottingham, UK

${ }^{3}$ School of Health, University of Northampton, UK

${ }^{4}$ University of Brighton, School of Health Professions, UK

${ }^{5}$ The National University of Ireland, Galway, Discipline of Podiatry, Galway, Ireland

${ }^{6}$ Glasgow Caledonian University, School of Health and Social Care, UK

${ }^{7}$ Hull York Medical School, UK Corresponding to: S Cockayne sarah.cockayne@york.ac.uk

Cite this as: $B M J$ 2011;342:d3271 doi:10.1136/bmj.d3271

\section{ABSTRACT}

Objective To compare the clinical effectiveness of cryotherapy versus salicylic acid for the treatment of plantar warts.

Design A multicentre, open, two arm randomised controlled trial.

Setting University podiatry school clinics, NHS podiatry clinics, and primary care in England, Scotland, and Ireland.

Participants 240 patients aged 12 years and over, with a plantar wart that in the opinion of the healthcare professional was suitable for treatment with both cryotherapy and salicylic acid.

Interventions Cryotherapy with liquid nitrogen delivered by a healthcare professional, up to four treatments two to three weeks apart. Patient self treatment with 50\% salicylic acid (Verrugon) daily up to a maximum of eight weeks.

Main outcome measures Complete clearance of all plantar warts at 12 weeks. Secondary outcomes were (a) complete clearance of all plantar warts at 12 weeks controlling for age, whether the wart had been treated previously, and type of wart, (b) patient self reported clearance of plantar warts at six months, $(c)$ time to clearance of plantar wart, $(d)$ number of plantar warts at 12 weeks, and (e) patient satisfaction with the treatment. Results There was no evidence of a difference between the salicylic acid and cryotherapy groups in the proportions of participants with complete clearance of al plantar warts at 12 weeks (17/119 (14\%) v 15/110 (14\%), difference $0.65 \%(95 \% \mathrm{Cl}-8.33$ to 9.63$), \mathrm{P}=0.89)$. The results did not change when the analysis was repeated but with adjustment for age, whether the wart had been treated previously, and type of plantar wart or for patients' preferences at baseline. There was no evidence of a difference between the salicylic acid and cryotherapy groups in self reported clearance of plantar warts at six months (29/95 (31\%) v 33/98 (34\%), difference $-3.15 \%$ ( -16.31 to 10.02$), \mathrm{P}=0.64)$ or in time to clearance (hazard ratio 0.80 (95\% Cl 0.51 to 1.25$)$, $\mathrm{P}=0.33$ ). There was also no evidence of a difference in the number of plantar warts at 12 weeks (incident rate ratio 1.08 (0.81 to 1.43$), \mathrm{P}=0.62$ ).

Conclusions Salicylic acid and the cryotherapy were equally effective for clearance of plantar warts. Trial registration Current Controlled Trials ISRCTN18994246, National Research Register N0484189151.

\section{INTRODUCTION}

Verrucae (or plantar warts) are extremely common, being experienced by most people at some time during their lives. Studies that have examined the prevalence of warts or verrucae have produced a wide range of estimates-from $0.84 \%$ in the US, ${ }^{1} 3.3 \%$ to $4.7 \%$ in the $\mathrm{UK}^{23}$ and up to $24 \%$ in $16-18$ year olds in Australia. ${ }^{4}$ Although most plantar warts will spontaneously disappear without treatment, ${ }^{56}$ many patients seek treatment for a variety of reasons, including discomfort or because they are prevented from doing sports and other activities of daily living. Almost two million people in England and Wales see their general practitioner for the treatment of cutaneous warts each year, at an annual cost of at least $£ 40 \mathrm{~m} .^{7}$ There are many different treatments for cutaneous warts, including cryotherapy, topically applied treatments, surgical curettage, and complementary and alternative therapies. The most commonly prescribed treatments for plantar warts are cryotherapy with liquid nitrogen and topical salicylic acid. ${ }^{8}$

A Cochrane systematic review assessed the effects of different local treatments of cutaneous, non-genital warts and highlighted considerable uncertainty around the optimal treatment. ${ }^{6}$ The best available evidence was for topical treatments containing salicylic acid (of various strengths). These preparations were significantly better than placebo. Data pooled from five placebo controlled trials showed a cure rate of 117/160 (73\%) compared with 78/162 (48\%) in controls. Evidence for the effectiveness of cryotherapy was limited: the review found two trials comparing cryotherapy with salicylic acid, and one comparing it with duct 
tape. These trials showed no significant difference in efficacy for the compared treatments. More recently, a head to head trial compared salicylic acid and cryotherapy in a primary care setting in the Netherlands. This trial found that cryotherapy was significantly better than salicylic acid for the treatment of hand warts, but that there was no significant difference between treatments for plantar warts. ${ }^{9}$

The Cochrane systematic review further highlighted the lack of good quality evidence on which to inform clinical decision making. Of the 60 trials identified in the review, $46(77 \%)$ were classified as low quality, heterogeneity between the trials was high, and analyses were often inappropriate or misleading. A major conclusion from the review was that a trial comparing topical salicylic acid with cryotherapy was urgently needed. We therefore conducted a randomised controlled trial to compare the clinical and cost effectiveness of 50\% salicylic acid and cryotherapy with liquid nitrogen.

\section{METHODS}

This multicentre, two arm, randomised, controlled, open trial was carried out in 13 centres in the United Kingdom and one in the Republic of Ireland.

\section{Study population}

Participants were eligible for the study if they were aged 12 years or over and had a plantar wart (verruca) that, in the opinion of a healthcare professional, was suitable for treatment with both salicylic acid and cryotherapy. Participants were excluded from the study if they had impaired healing (such as from diabetes or peripheral vascular disease); were immunosuppressed (such as agammaglobulinaemia) or were taking immunosuppressant drugs (such as oral corticosteroids); had neuropathy; were receiving renal dialysis; had cold intolerance (such as Raynaud's syndrome or cold urticaria); had any of the following conditions (blood dyscrasias of unknown origin, cryoglobulinaemia, cryofibrinogenaemia, collagen or autoimmune disease); were unable to give informed consent; or were currently in a trial evaluating other treatments for their plantar wart.

\section{Recruitment and randomisation of participants}

Participants were recruited between November 2006 and January 2010 (end of extended recruitment period) from university podiatry school clinics, NHS podiatry clinics, and primary care. Eligible participants gave written informed consent. They were then randomised equally to receive cryotherapy with liquid nitrogen or daily self treatment with an over the counter $50 \%$ salicylic acid treatment.

Randomisation was performed by a member of the research team either telephoning an independent, secure, remote, telephone randomisation service (York Trials Unit) or accessing a secure online web randomisation programme, thereby concealing treatment allocation until the moment of randomisation. Randomisation was simple (that is, it was not restricted in any way such as by stratification or blocked allocation with the allocation sequence being computer generated).

\section{Intervention}

\section{Cryotherapy}

Participants randomised to cryotherapy with liquid nitrogen received a maximum of four treatments given two to three weeks apart by a healthcare professional. The liquid nitrogen was applied with a spray (method of choice if available) or a probe. The treatment was delivered according to the site's usual practice (such as debridement before treatment, masking of the surrounding area, and padding after treatment). On advice from the trial steering committee, sites were advised that the first treatment should be a "gentle freeze" in order to ensure the participant could tolerate the treatment.

\section{Salicylic acid}

Participants randomised to self treatment with 50\% salicylic acid (Verrugon, William Ransom and Son) were instructed how to apply the treatment according to the manufacturer's instructions by the treating healthcare professional. Patients were directed to fix the adhesive ring with the hole over the verruca and to squeeze a little ointment into the hole and directly on to the verruca. The backing paper from the plaster was then removed and the plaster applied to cover the ring completely. The plaster was then sealed into position. The treatment was repeated daily after gently pumicing or filing off the dead part of the verruca for a maximum of eight weeks.

\section{Outcome measurements \\ Primary outcome}

The primary outcome was complete clearance of all plantar warts at 12 weeks after randomisation. Clearance of plantar wart was defined as the restoration of normal skin on close inspection. Digital photographs of the plantar wart(s) were taken at baseline and at the outcome assessment. The photographs were assessed by two assessors who were blind to treatment allocation. They independently assessed the photographs for each participant to determine whether the plantar wart had cleared. Any discrepancies were referred to a third assessor. If no photographs were available for a participant, however, then the blinded outcome assessment undertaken at the site was used. If neither of these were available for a participant, the patient's self reported outcome recorded in the week 12 patient questionnaire or on the "Verrucae gone form" was used.

\section{Secondary outcomes}

Secondary outcomes included $(a)$ complete clearance of all plantar warts at 12 weeks after controlling for age, whether the plantar wart had been treated previously, and type of wart and $(b)$ a second model to explore the effect of patient preferences, $(c)$ with clearance of plantar wart at six months, $(d)$ number of warts at 12 weeks, (e) time to clearance of wart, $(f)$ patient satisfaction with the treatment, and $(g)$ adverse events. Questionnaires 
were administered by post or were completed on line at one, three, and 12 weeks and six months.

\section{Sample size \\ The Cochrane systematic review found only one small trial directly comparing the effectiveness of a chemical treatment, salicylic acid, with cryotherapy in patients with warts on their feet alone. ${ }^{6}$ This poor quality study found a $58 \%$ cure rate among the patients allocated to cryotherapy, compared with $41 \%$ among those treated with salicylic acid. This difference of $17 \%$ was not sta- tistically significant. The overall cure rates from this study are smaller than those observed in two placebo controlled trials of salicylic acid, both of which reported cure rates of $85 \%$ for active treatment, possi- bly because more resistant verrucae were included in the study comparing cryotherapy with salicylic acid This trial was a superiority study of cryotherapy com- pared with salicylic acid and was powered to show a $15 \%$ difference in effectiveness. In order to give $80 \%$ power ( $5 \%$ two sided significance) to show a difference in cure rates of $70 \%$ for salicylic acid versus $85 \%$ for cryotherapy at 12 weeks, a sample size of 120 patients in each treatment group was required, or 133 patients in each group after allowing for 10\% attrition (266 patients in total).}

\section{Statistical analysis}

All analyses were conducted on an intention to treat basis, including all patients in the groups to which

\section{Assessed for eligiblity $(n=284)$}

\begin{tabular}{|c|c|}
\hline & $\begin{array}{l}\text { Excluded }(n=44)^{\star} \text { : } \\
\text { Not eligible for both treatments }(n=12) \\
\text { Under } 12 \text { years of age }(n=2) \\
\text { Unable to give informed consent }(n=1) \\
\text { Impaired healing }(n=6) \\
\text { Taking immunosuppressant drugs }(n=3) \\
\text { Involved in another trial }(n=3) \\
\text { Other reasons }(n=25)\end{array}$ \\
\hline \multicolumn{2}{|c|}{ Randomised $(n=242)$} \\
\hline & $\begin{array}{l}\text { Randomised in error }(n=2) \text { : } \\
\text { Cryotherapy }(n=1) \\
\text { Salicylic acid }(n=1)\end{array}$ \\
\hline Allocated to cryotherapy $(n=117)$ & Allocated to salicylic acid $(n=123)$ \\
\hline$\downarrow$ & $\downarrow$ \\
\hline $\begin{array}{l}\text { Lost to questionnaire follow-up }(n=13) \text { : } \\
\text { Week } 1(n=5) \\
\text { Week } 3(n=0) \\
\text { Week } 12(n=5) \\
\text { Withdrawn from study }(n=3) \dagger\end{array}$ & $\begin{array}{l}\text { Lost to questionnaire follow-up }(n=16) \text { : } \\
\text { Week } 1(n=8) \\
\text { Week } 3(n=3) \\
\text { Week } 12(n=5) \\
\text { Withdrawn from study }(n=0)\end{array}$ \\
\hline$\downarrow$ & $\downarrow$ \\
\hline $\begin{array}{l}\text { Primary outcome analysis at } 12 \text { weeks }(n=110) \\
\text { Missing data }(n=4)\end{array}$ & $\begin{array}{l}\text { Primary outcome analysis at } 12 \text { weeks }(n=119) \\
\text { Missing data }(n=4)\end{array}$ \\
\hline$\downarrow$ & $\downarrow$ \\
\hline $\begin{array}{l}\text { Analysed at } 6 \text { months }(n=98) \\
\text { Missing data }(n=6)\end{array}$ & $\begin{array}{l}\text { Analysed at } 6 \text { months }(n=95) \\
\text { Missing data }(n=12)\end{array}$ \\
\hline
\end{tabular}

* Patients could be excluded for more than one reason

† Withdrawals because healthcare practitioner on sick leave and no other member of the research team available to run the study

Flow of participants through the EVerT trial they were randomised. Analyses were conducted in SAS version 9.2 (SAS Institute, NC, USA) and SPSS version 17.0.2 (SPSS) using two sided significance tests at the $5 \%$ significance level for the primary outcome measure and $1 \%$ significance level for secondary outcome measures.

\section{Primary analysis}

The primary analysis compared the clearance rate of all plantar warts at 12 weeks between the two randomised groups using a $\chi^{2}$ test. Cohen's $\kappa$ measure of inter-rater agreement was used to assess agreement between the two assessors of the photographs for clearance.

\section{Secondary analysis}

A logistic regression model was used to adjust the primary analysis for important prognostic variables (age, whether the plantar wart had been previously treated, and type of wart). We also extended the primary outcome model to explore the effect of patient preferences by including preference and an interaction term between preferred treatment and randomised treatment. The complete clearance of all plantar warts at six months was analysed in the same way as the primary outcome with adjustments for the same covariates. We used negative binomial regression to compare the number of plantar warts at 12 weeks between the two treatment groups with adjustment for the number of plantar warts at baseline. These models are used to estimate the number of occurrences of an event when the event has Poisson variation with over-dispersion.

The time to clearance of plantar warts was derived as the number of days from randomisation until the date of clearance, as detailed from the participant's self reported questionnaire. Participants whose plantar warts had not cleared were treated as censored, and we calculated their duration in the trial from their date of trial exit, date of last available assessment, or the 183 days to trial cessation, as appropriate. A Cox proportional hazards model was used to compare the time to clearance of plantar warts between the two groups adjusting for the same covariates as for the primary outcome. The Cox proportional hazard assumption was tested globally by the correlation of Schoenfeld residuals and survival time (or ranked survival time) and separately for each covariate through the correlation of Schoenfeld scaled residuals and survival time (or ranked survival time). Non-significant correlation indicates that there is not enough evidence that the proportional hazard assumption has been violated.

Data on patient satisfaction with the treatment and adverse events were summarised by treatment group but no statistical analyses were performed.

\section{RESULTS}

Between November 2006 and January 2010, 284 individuals were screened as potential participants, and $242(85 \%)$ were randomised-124 to salicylic acid and 118 to cryotherapy. The figure shows the flow of participants through the trial, and table 1 summarises 
Table 1|Baseline characteristics of 240 participants with plantar warts assigned to treatment with cryotherapy or salicylic acid

\begin{tabular}{lcc}
$\begin{array}{l}\text { Characteristics } \\
\text { No (\%) of each sex: }\end{array}$ & $\begin{array}{c}\text { Cryotherapy } \\
(\mathrm{n}=117)\end{array}$ & $\begin{array}{c}\text { Salicylic acid } \\
(\mathrm{n}=123)\end{array}$ \\
\hline Female & $86(74)$ & $73(59)$ \\
\hline Male & $31(26)$ & $50(41)$ \\
\hline Age (years): & $30.1(15.7)$ & $30.2(16.4)$ \\
\hline Mean (SD) & $24.3(12.2-75.3)$ & $23.2(12.0-70.6)$ \\
\hline Median (range) & & \\
\hline
\end{tabular}

their baseline characteristics. Table 2 summarises the baseline characteristics of their plantar warts.

\section{Treatment details}

Table 3 summarises the cryotherapy treatment details reported by the treating healthcare professional. The mean number of visits to the clinic or general practice for cryotherapy was 3.6 , with a mean duration between visits of 18.3 days. Table 4 summarises the treatment

Table 2 | Baseline characteristics of plantar warts (verrucae) among 237 participants assigned to treatment with cryotherapy or salicylic acid*. (Values are numbers (percentages) unless stated otherwise)

\begin{tabular}{|c|c|c|}
\hline Characteristics & $\begin{array}{c}\text { Cryotherapy } \\
(n=114)\end{array}$ & $\begin{array}{c}\text { Salicylic acid } \\
\quad(n=123)\end{array}$ \\
\hline No of plantar warts per participant: & $(n=106)$ & $(n=119)$ \\
\hline Mean (SD) & $4.0(6.6)$ & $3.4(3.6)$ \\
\hline Median (range) & $2.0(1.0-55.0)$ & $2.0(1.0-20.0)$ \\
\hline Duration of wart (months): & $(n=108)$ & $(n=119)$ \\
\hline Mean (SD) & $25.0(24.9)$ & $26.9(24.5)$ \\
\hline Median (range) & $14.5(0.9-144.0)$ & $20(1.5-130.0)$ \\
\hline Type of plantar wart: & $(n=108)$ & $(n=120)$ \\
\hline Mosaic & $29(26.9)$ & $21(17.5)$ \\
\hline Non-mosaic & $79(73.1)$ & $99(82.5)$ \\
\hline Previous treatment: & $(n=114)$ & $(n=123)$ \\
\hline Yes & $89(78)$ & $96(78)$ \\
\hline No & $25(22)$ & $27(22)$ \\
\hline Type of previous treatment†: & $(n=89)$ & $(n=96)$ \\
\hline Self treatment & $81(91)$ & $82(85)$ \\
\hline Podiatrist or chiropodist & $24(27)$ & $29(30)$ \\
\hline General practitioner & $30(34)$ & $43(45)$ \\
\hline Trial investigating verruca treatments & $0(0)$ & $2(2)$ \\
\hline Other & $6(7)$ & $8(8)$ \\
\hline Reasons for seeking plantar wart treatment†: & $(n=114)$ & $(n=123)$ \\
\hline Painful & $68(60)$ & $71(58)$ \\
\hline Unable to go swimming & $29(25)$ & $41(33)$ \\
\hline Unable to participate in other sports & $18(16)$ & $28(23)$ \\
\hline Other & $46(40)$ & $59(48)$ \\
\hline Pain intensity: & $(n=106)$ & $(n=119)$ \\
\hline Not at all & $44(39)$ & $44(36)$ \\
\hline A little bit & $37(33)$ & $34(28)$ \\
\hline Moderately & $20(18)$ & $22(18)$ \\
\hline Quite a lot & $10(9)$ & $18(15)$ \\
\hline Extremely & $2(2)$ & $4(3)$ \\
\hline
\end{tabular}

*Some data missing for certain characteristics.

†More than one category could be checked. details for the salicylic acid group. Participants applied salicylic acid on a mean of 6.3 days in week 1 and 5.4 days in week 3 .

\section{Primary outcome}

In total, 229 participants had a response for whether there was complete clearance of all plantar warts at 12 weeks after randomisation, with 206 (90\%) having a blinded outcome assessment: 159 (69\%) had a blinded outcome assessment from a digital photograph (cryotherapy $=80$, salicylic acid=79), $47(21 \%)$ had a blinded outcome assessment from a healthcare professional (cryotherapy $=22$, salicylic acid $=25)$, four $(2 \%)$ had an unblinded outcome assessment from a healthcare professional (cryotherapy $=1$, salicylic acid $=3$ ), and $19(8 \%)$ had patient self assessment (cryotherapy $=7$, salicylic acid=12). Overall, 32 of the 229 (14\%) had complete clearance of all plantar warts at 12 weeks, corresponding to $17 / 119(14 \%)$ patients in the salicylic acid group and 15/110 (14\%) patients in the cryotherapy group, with no significant difference between the two groups (difference $0.65 \%$ (95\% confidence interval -8.33 to 9.63$), \mathrm{P}=0.89$ ).

Cohen's $\kappa$ measure, used to assess the agreement between the two assessors of the photographs, was estimated to be 0.45 (95\% confidence interval 0.35 to $0.55)$, which indicates a moderate level of agreement. The assessors disagreed in 51 cases: in three cases the disagreement was between whether the plantar wart was "cleared" or "not cleared," in five cases it was between "cleared" and "unable to assess," and in the remaining 43 cases it was between "not cleared" and "unable to assess." This might lead to an underestimation of the clearance rate, but as the assessment was blind to treatment allocation it is unlikely to lead to a difference between the two treatments groups.

\section{Secondary outcome}

The primary analysis was repeated after controlling for age, whether the plantar warts had been previously treated (yes or no), and the type of plantar wart (mosaic or non-mosaic, where a mosaic was defined as a group of numerous, closely aggregated plantar warts on the sole of the foot). Logistic regression analysis showed no evidence of a difference between the salicylic acid and cryotherapy groups (odds ratio 0.96 (95\% confidence interval 0.44 to 2.11 ), $\mathrm{P}=0.92$ which favours the salicylic group).

We received patient self reported data on presence or absence of plantar warts at six months from 193 participants. Overall, 62 (32\%) of the patients had complete clearance of all plantar warts at six months$29 / 95(31 \%)$ of patients in the salicylic acid group and $33 / 98(34 \%)$ in the cryotherapy group, with no significant difference between the groups (difference $-3.15 \%$ $(-16.31$ to 10.02$), \mathrm{P}=0.64)$. The findings from the adjusted analysis were similar to those of the unadjusted analysis (odds ratio 1.17 (0.62 to 2.21 ), $\mathrm{P}=0.62$ ).

The median number of plantar warts at 12 weeks in the salicylic acid group was two (range 0-20) and in the cryotherapy group was one (0-40), and no significant 
Table $3 \mid$ Details of cryotherapy received by 109 patients for treatment of plantar warts. (Values are numbers (percentages) unless stated otherwise)

\begin{tabular}{lc} 
Details & $\begin{array}{c}\text { Cryotherapy group } \\
(\mathrm{n}=109)\end{array}$ \\
No of visits: & $3.6(0.7)$ \\
\hline Mean (SD) & $4.0(1.0-5.0)$ \\
\hline Median (range) & $18.3(6.8)$ \\
\hline Duration between visits (days): & $15.5(9.7-52.5)$ \\
\hline Mean (SD) & $1.6(0.7)$ \\
\hline Median (range) & $1.5(0.3-4.3)$ \\
\hline Mean (SD) & $10.9(8.6)$ \\
\hline Median (range) & $9.5(2.0-60.0)$ \\
\hline Duration of each application (seconds): & $103(94)$ \\
\hline Mean (SD) & $10(9)$ \\
\hline Median (range) &
\end{tabular}

difference between the two groups (incident rate ratio 1.08 (0.81 to 1.43 ), $\mathrm{P}=0.62$ ). The number of plantar warts at the start of the study was not found to be an important predictor of outcome (odds ratio 0.81 (0.65 to 1.01$)$ ).

We compared the time to clearance of the plantar warts between the two groups adjusting for the same covariates as above (age, previous treatment, and type of wart). There was no evidence of a difference in the time to clearance between cryotherapy and salicylic acid in the Cox proportional hazards model (hazard ratio 0.80 ( 0.51 to 1.25$), \mathrm{P}=0.33$ ). The proportional hazard assumption was tested and not violated, either separately for each covariate (cryotherapy $v$ salicylic acid $(\mathrm{P}=0.3107)$, age $(\mathrm{P}=0.9508)$, type of plantar wart $(\mathrm{P}=0.7024)$, previous treatment $(\mathrm{P}=0.4456))$ or globally $(\mathrm{P}=0.8208)$. Hence all hazard ratios were assumed constant during the follow-up period.

\section{Satisfaction with treatment}

Patients were asked to rate their satisfaction with their treatment on a five point scale from "very happy" to

Table 5 Patient satisfaction with cryotherapy or salicylic acid treatment for plantar warts at 1,3 , and 12 weeks after randomisation. (Values are numbers (percentages) of patients)

\begin{tabular}{|c|c|c|c|c|c|}
\hline \multirow[b]{2}{*}{ Time and treatment } & \multicolumn{5}{|c|}{ Satisfaction with treatment } \\
\hline & Very unhappy & Unhappy & Indifferent & Happy & Very happy \\
\hline \multicolumn{6}{|l|}{ Week 1: } \\
\hline Cryotherapy $(n=101)$ & $6(6)$ & $1(1)$ & $26(26)$ & $43(43)$ & $25(25)$ \\
\hline Salicylic acid $(n=107)$ & $1(1)$ & $5(5)$ & $32(30)$ & $54(50)$ & 15 (14) \\
\hline \multicolumn{6}{|l|}{ Week 3: } \\
\hline Cryotherapy $(n=104)$ & $10(10)$ & $0(0)$ & $18(17)$ & $48(46)$ & $28(27)$ \\
\hline Salicylic acid $(n=104)$ & $4(4)$ & $8(8)$ & $29(28)$ & $46(44)$ & $17(16)$ \\
\hline \multicolumn{6}{|l|}{ Week 12: } \\
\hline Cryotherapy $(n=91)$ & $7(8)$ & $5(5)$ & $23(25)$ & $30(33)$ & $26(29)$ \\
\hline Salicylic acid $(n=98)$ & $10(10)$ & $21(21)$ & $27(28)$ & $25(26)$ & 15 (15) \\
\hline
\end{tabular}

Percentages may not add up to 100 because of rounding.
Table $4 \mid$ Details of self treatment with salicylic acid by 108 patients for treatment of plantar warts. (Values are numbers (percentages) unless stated otherwise)

\begin{tabular}{lc} 
Detail & $\begin{array}{c}\text { Salicylic acid group } \\
(\mathrm{n}=108)\end{array}$ \\
No of tubes dispensed: & \\
\hline 1 & $82(76)$ \\
\hline 2 & $26(24)$ \\
\hline Weight of salicylic acid used $(\mathrm{g}):$ & $(\mathrm{n}=58)$ \\
\hline Mean (SD) & $2.89(2.2)$ \\
\hline Median (range) & $2.4(0.06-9.3)$ \\
\hline No of times salicylic acid applied & \\
\hline in past 7 days: & $(\mathrm{n}=106)$ \\
\hline Week 1: & $6.3(1.5)$ \\
\hline Mean (SD) & $7.0(0-7)$ \\
\hline Median (range) & $(\mathrm{n}=103)$ \\
\hline Week 3: & $5.4(2.8)$
\end{tabular}

"very unhappy" on a postal or web based questionnaire at one, three, and 12 weeks (see table 5). There was a significant association between patient satisfaction with treatment and randomised treatment at each of the three time points (Fisher's exact test $\mathrm{P}=0.0336$ for week $1, \mathrm{P}=0.0020$ for week $3, \mathrm{P}=0.0065$ for week 12). At week 1 , more patients were happy with salicylic acid than cryotherapy but also more patients were very happy with cryotherapy than salicylic acid. At week 3, more patients were unhappy with salicylic acid than with cryotherapy (none), and more patients were very happy with cryotherapy than salicylic acid. At week 12 , more patients were unhappy with salicylic acid than with cryotherapy, and more were very happy with cryotherapy than with salicylic acid. This is not expected if satisfaction with treatment was independent of treatment randomised.

\section{Adverse events}

Data on adverse events were collected by the treating healthcare professional, by review of patients' medical notes by the trial coordinator at site monitoring visits, or by review of participant follow-up questionnaires. A total of 28 adverse events were reported in 19 participants. Of these, one event (a ruptured Achilles tendon requiring hospitalisation) was classed as serious and unrelated to the trial treatment (salicylic acid). The remaining 27 were assessed as non-serious, 13 of which were in the salicylic acid group and 14 in the cryotherapy group. Of the 13 non-serious adverse events in the salicylic acid group, nine were unrelated to the trial treatment and four were unlikely to be related to the treatment. In the cryotherapy group, there were two treatment related, non-serious adverse events in two participants. Both participants developed a blister that was larger than expected in routine practice. The remaining 12 adverse events in the cryotherapy group were either unrelated to the trial treatment (7) or unlikely to be related (5). 


\section{DISCUSSION}

\section{Key findings}

We compared the clinical effectiveness of cryotherapy with liquid nitrogen and $50 \%$ salicylic acid for the treatment of plantar warts and found no evidence of a difference in effectiveness. However, cryotherapy was associated with higher costs per cured patient, which leads to salicylic acid being a more attractive treatment.

\section{Comparison with other studies}

Our results confirm the findings of the two published studies comparing cryotherapy with salicylic acid ${ }^{1011}$ and the results from a more recent Dutch primary care study ${ }^{9}$ which compared cryotherapy with salicylic acid or a combination of salicylic and lactic acid for the treatment of plantar and hand warts. These studies also showed no evidence that cryotherapy was more effective than salicylic acid (alone or in combination with lactic acid).

Our trial, however, does differ from the previous studies in respect to the cure rate. Our overall cure rate was $14 \%(32 / 229)$, whereas cure rates in the earlier studies ranged from about $30 \%{ }^{9}$ to $68 \%,{ }^{11}$ at least twice the rate we observed. This difference in cure rate could be attributed to different populations recruited to the study. For example, Bunney et al included only patients with hand warts, ${ }^{10}$ while Steele and Irwin excluded mosaic warts, ${ }^{11}$ which are generally regarded as more resistant to treatment. They also excluded patients with five or more lesions, lesions outside an average diameter of 3-9 $\mathrm{mm}$, and patients who had self treated within the previous month. In our study $22 \%$ of participants had a mosaic wart, $17 \%$ had more than five verrucae, and most had already tried other treatments. There was also a difference in the age of the populations. Patients were younger in the studies by Bruggink et $\mathrm{al}^{9}$ and Steele and Irwin, ${ }^{11}$ with $59 \%$ of participants under the age of 16 in the latter study compared with $17 \%$ in our study. The median age of patients in Bruggink et al's study was 15 years (interquartile range 7-39) for cryotherapy patients and 13 (7-31) years for salicylic acid patients compared with median ages of 24 and 23 respectively in our study.

\section{Strengths and limitations of the study}

The strengths of this study include adequate randomisation, allocation concealment, blinded outcome assessment, and intention to treat analysis, all of which minimises the risk of bias. A further strength is that this was a large pragmatic study that recruited patients from 14 centres across England, Scotland, and Ireland from podiatry clinics, general practices, and from the community. The participants had longstanding plantar warts, most of which had been self treated. This is typical of the characteristics of patients presenting to healthcare professionals for treatment, so we can be confident that these results are broadly generalisable and that the study has external validity across the UK and Ireland.

Our study has several potential limitations. Firstly, our sample size was powered to show a $15 \%$ difference in effectiveness, with cure rates of $70 \%$ for the salicylic acid treatment and $85 \%$ for the cryotherapy. However, the overall cure rate achieved in this trial was much lower at $14 \%$ with a $0.65 \%$ difference in cure rates.

Secondly, it has been suggested that a six month follow-up is a more realistic time point at which to assess the success of treatments of cutaneous warts than shorter time frames. ${ }^{6}$ Assessment at six months not only allows for the fact that the human papilloma virus may remain dormant within epithelial cells without visible disease but would also allow time for any response mediated by the immune system to be observed. However, we chose to assess the primary outcome in our study at 12 weeks because we know many plantar warts spontaneously resolve, so the important clinical question is whether treating plantar warts makes them go sooner. We considered that 12 weeks is a reasonable time frame in which to expect a treatment for plantar warts to be effective. In this study neither treatment was very effective with only $17 / 119(14 \%)$ in the salicylic acid group and 15/110 $(14 \%)$ in the cryotherapy group having complete clearance of plantar warts at 12 weeks, but the lack of a no treatment arm in this study means we cannot compare these cure rates with the natural resolution rate.

Finally, participants allocated to the salicylic acid group were advised to self treat for a maximum of eight weeks, but we collected patient self reported data on adherence to treatment only at one and three weeks after randomisation. Self reported adherence at these times was reasonably high, with participants applying salicylic acid on a mean of 6.3 days and 5.4 days out of seven respectively. However, data collected by the healthcare professionals about the total amount of acid applied during the course of the trial (mean amount applied $2.8 \mathrm{~g}$ ) could suggest a lower adherence for the treatment period overall. For some deep seated plantar warts, this might not have been sufficient to clear the wart.

\section{Conclusion and implications of results}

Here we report the results of a high quality trial evaluating two common treatments for plantar warts. We found no evidence that cryotherapy is more effective than patient self treatment with 50\% salicylic acid for the treatment of plantar warts. If we add our trial data into a meta-analysis, we find the odds ratio for cure is 1.07 (95\% confidence interval 0.63 to 1.79$)$, indicating that the odds of clearance of plantar warts is similar with either treatment.

However, the only salicylic acid preparation we evaluated was at a $50 \%$ concentration, which is not the most commonly used. This concentration of salicylic acid is similar to that often used by podiatrists to treat plantar warts and is often viewed as a second line treatment. Typically, first line treatments in the UK are weaker preparations of 15\%-26\% salicylic acid. The Cochrane systematic review identified only one trial that compared two different concentrations of salicylic acid. ${ }^{6}$ This trial found no evidence of a difference in the cure rates between the two treatments, but, 


\section{WHAT IS ALREADY KNOWN ON THIS TOPIC}

Most plantar warts spontaneously disappear without treatment, but patients may seek treatment if the warts are painful or because they are being prevented from doing sports and other activities of daily living

There are many different ways to treat plantar warts, but the evidence base to inform clinical decision making is poor

\section{WHAT THIS STUDY ADDS}

There is no evidence to suggest that cryotherapy with liquid nitrogen was more clinically effective than patient self treatment with $50 \%$ salicylic acid in clearing plantar warts

Since cryotherapy is substantially more expensive than treatment with salicylic acid, this makes salicylic acid the more cost effective treatment
Assessment Programme and refined the protocol. SC acts as guarantor for the paper. DT and IW were chief investigators and oversaw the study. SC and $\mathrm{KH}$ were the trial coordinators, and GT was the trial support officer. Gill Worthy, SJ, and CH designed the clinical analysis. CH oversaw the conduct of the analysis. SJ and ARK conducted the clinical analysis. ES designed and undertook the economic analysis. The writing team consisted of SC, $\mathrm{KH}, \mathrm{CH}, \mathrm{ES}, \mathrm{KT}$, and ARK, who drafted the report. GD, CM, FH, SJ, DT, IW, and GT commented on the report.

Funding: This project was funded by the UK National Institute for Health Research, Health Technology Assessment Programme (project No 05/ 513/02) and will be published in full in the journal Health Technology Assessment. The views and opinions expressed are those of the authors and do not necessarily reflect those of the Department of Health. William Ransom and Son supplied the $50 \%$ salicylic acid (Verrugon) at no cost, and BOC provided one site with liquid nitrogen storage equipment at reduced cost. These manufacturers had no role in the design of the trial or in the collection, analysis, and interpretation of data.

Competing interests: All authors have completed the ICMJE uniform disclosure form at www.icmje.org/coi_disclosure.pdf (available on request from the corresponding author) and declare: SC, KH, SJ, GT, KT, $\mathrm{MC}, \mathrm{FH}, \mathrm{NM}$, and DT received proportions of their salaries from the Health Technology Assessment grant in order to conduct the study; no financial relationships with any organisations that might have an interest in the submitted work in the previous three years; and no other relationships or activities that could appear to have influenced the submitted work. Ethical approval: This study was approved by Trent Multicentre Research Ethics Committee, Galway Research Ethics Committee, and local ethics committees, Medicines and Healthcare products Regulatory Agency, Irish Medicines Board, and local research and development trusts. Data sharing: No additional data available.

1 Johnson ML, Roberts J. Skin conditions and related need for medical care among persons 1-74 years. US Department of Health Education and Welfare, 1978: 1-26.

2 Rea JN, Newhouse ML, Halil T. Skin disease in Lambeth. A community study of prevalence and use of medical care. BrJ Prev Soc Med 1976;30:107-14.

general practitioners, and practice nurses for recruiting participants to the study and completing the trial documentation; the principal investigators at each site for coordinating participant recruitment; members of the tria steering committee (Sam Gibbs (independent chair), Jill Mollison, Elaine Thomas, and Wesley Vernon); and members of the data monitoring and ethics committee (Anne-Maree Keenan (independent chair), Matthew Hankins, and Katharine Speaks for overseeing the study. We also thank Jil Hall, Jude Watson, and Farina Hashmi, who undertook the blinded outcome assessment of the digital photographs and Gill Worthy for providing statistical advice.

The EVerT collaborators (current and past) are Peter Arthur, Lucy Bellas, Beverly Brown, Lynne Bryan, Amanda Clark, Andrew Clarke, Michae Concannon, Beryl Cooling, Dawn Curruthers, Chris Davies, Gary Denby, Sean Dinneen, Diane Exley, Lisa Farndon, Simon Gazeley, Elizabeth Green, Stephanie Haughy, Julia Haswell, Christine Hearmon, Christine Howell, Arthur Kang'ombe, Jamil Karolia, Susan Kitchener, Phillip LeDune, Susan Lightfoot, Maria Madigan, Julie Mandehzadeh, Rina Miah, Caroline McIntosh, Christine Northern, Frances Price, Julie Poland, Ilan Rajap, Jayne Robinson, Julie Robinson, Raymond Skinner, Deborah Turner, Susan Walton, Linda White, Catriona Williams, who were members of the research team at each site and recruited participants to the study; Fiona Aitken and Helen Hill from the Medicines for Children Local Research Network; and Kate Biscomb and Kate Wyer from the Primary Care Research Network.

Contributors: DT and jill Hall wrote the original protocol. SC, MC, FH, NM, DT, and KT were co-applicants on the application to Health Technology
3 Williams HC, Pottier A, Strachan D. The descriptive epidemiology of warts in British schoolchildren. Br J Dermatol 1993;128:504-11.

4 Kilkenny M, Merlin K, Young R, Marks R. The prevalence of common skin conditions in Australian school students: 1. Common, plane and plantar viral warts. BrJ Dermatol 1998;138:840-5.

5 Massing AM. Natural history of warts. Arch Dermatol 1963;87:306-10.

6 Gibbs S, Harvey I. Topical treatments for cutaneous warts. Cochrane Database Syst Rev 2006;3:CD001781.

7 Thomas KS, Keogh-Brown MR, Chalmers JR, Fordham RJ, Holland RC, Armstrong SJ, et al. Effectiveness and cost-effectiveness of salicylic acid and cryotherapy for cutaneous warts: an economic decision model. Health Technol Assess 2006;10:iii, ix-87.

8 Schofield I, Grindlay D, Williams HC. Skin conditions in the UK: a health care needs assessment. Centre of Evidence Based Dermatology, University of Nottingham, 2009.

9 Bruggink SC, Gussekloo J, Zaaijer K, Assendelft WJJ, Berger MY, Koes BW, et al. Warts: cryotherapy, salicylic acid or expectantly awaiting? A randomised controlled trial. J Invest Dermatol 2008;128:8.

10 Bunney M, Nolan M, Williams D. An assessment of methods of treating viral warts by comparative treatment trials based on a standard design. Br J Dermatol 1976;94:667-79.

11 Steele K, Irwin W. Liquid nitrogen and salicylic/lactic acid paint in the treatment of cutaneous warts in general practice. J $R$ Coll Gen Pract 1988;38:256.

Accepted: 20 April 2011 